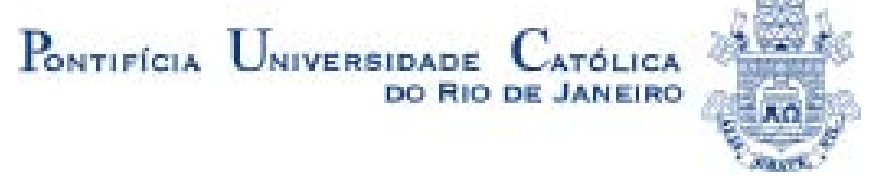

Rogério Odivan Brito Serrão

\title{
Operacionalização da flexibilidade de manufatura: análise das influências do relacionamento produtor-fornecedor
}

Tese de Doutorado

Tese apresentada ao Programa de Pós-graduação em Engenharia de Produção do Departamento de Engenharia Industrial da PUC-Rio como parte dos requisitos parciais para obtenção do título de Doutor em Engenharia de Produção.

Orientador: Paulo Roberto Tavares Dalcol

Rio de Janeiro

Setembro de 2005 


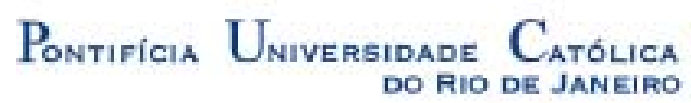

Rogério Odivan Brito Serrão

\section{Operacionalização da flexibilidade de manufatura:}

análise das influências do relacionamento produtor-fornecedor

Tese apresentada como requisito parcial para obtenção do título de Doutor pelo Programa de Pós-Graduação em Engenharia Industrial da PUC-Rio. Aprovada pela Comissão Examinadora abaixo assinada.

Prof. Paulo Roberto Tavares Dalcol

Orientador

Departamento de Engenharia Industrial - PUC-Rio

Prof. Maria Ângela Campelo de Melo

Departamento de Engenharia Industrial - PUC-Rio

Prof. Luiz Felipe Roris Rodriguez Scavarda do Carmo

Departamento de Engenharia Industrial - PUC-Rio

Prof. Adriano Proença Universidade Federal do Rio de Janeiro - UFRJ

Prof. Ricardo Manfredi Naveiro

Programa de Engenharia de Produção - COPPE/UFRJ

Prof. José Eugenio Leal

Coordenador Setorial do Centro Técnico Científico - PUC-Rio

Rio de Janeiro, 19 de setembro de 2005 
Todos os direitos reservados. É proibida a reprodução total ou parcial do trabalho sem autorização da universidade, do autor e do orientador.

\section{Rogério Odivan Brito Serrão}

Engenheiro Mecânico pela Universidade Federal do Pará (UFPA) em 1998. Mestre em Engenharia de Produção pela Pontifícia Universidade Católica do Rio de Janeiro (PUC-Rio) em 2001. Foi Visiting Researcher no Management Science Department da University of South Carolina (USC), Estados Unidos, em 2004. Possui experiência docente como Professor de Cursos de Pós-Graduação do IDHGE - CEFET/RJ e do Programa de Logística e Suprimentos PROCAE (Programa de Capacitação Executiva/UVA). Atualmente é Analista em Ciência \& Tecnologia no Instituto Brasileiro de Geografia e Estatística IBGE, na Coordenação de Recursos Materiais, no Rio de Janeiro/RJ. Autor de diversos artigos publicados em periódicos, congressos e simpósios nacionais e internacionais. Principais áreas de interesse e pesquisa: Gerência da Produção, Estratégia Empresarial, Logística Empresarial e Gerência de Projetos.

Ficha Catalográfica

Serrão, Rogério Odivan Brito

Operacionalização da flexibilidade de manufatura: análise das influências do relacionamento produtorfornecedor / Rogério Odivan Brito Serrão; orientador: Paulo Roberto Tavares Dalcol. - Rio de Janeiro: PUC, Departamento de Engenharia Industrial, 2005.

159 f.: il. ; $30 \mathrm{~cm}$

1. Tese (doutorado) - Pontifícia Universidade Católica do Rio de Janeiro, Departamento de Engenharia Industrial.

Inclui referências bibliográficas.

1. Engenharia Industrial - Teses. 2. Flexibilidade de manufatura. 3. Relacionamento produtor-fornecedor. 4. Desempenho da manufatura. I. Dalcol, Paulo Roberto Tavares. II. Pontifícia Universidade Católica do Rio de Janeiro. Departamento de Engenharia Industrial . III. Título. 
Ao meu Deus, Todo Poderoso, que me capacitou para ser vitorioso.

Aos meus pais Roque da Luz Serrão e Osmarina Brito Serrão pelo carinho e dedicação em minha formação como ser humano.

À Danielle Colares da Silva Serrão, minha esposa e incentivadora. 


\section{Agradecimentos}

Primeiramente, agradeço à Deus, que me abriu um horizonte fascinante e me mostrou a beleza da vida espiritual.

Expresso ainda meus sinceros agradecimentos a todos que contribuíram para a conclusão deste programa de doutorado na PUC-Rio.

Agradeço imensamente ao meu orientador Prof. Paulo Roberto Tavares Dalcol pelo incentivo em todos os momentos do programa, pela geração de oportunidades de aprendizado e crescimento como pesquisador, pelo profissionalismo na condução da orientação e pelos comentários sempre valiosos e precisos acerca da condução da pesquisa. Agradeço ainda pela amizade, companheirismo e suporte sempre presentes em momentos de adversidade.

Registro ainda meus agradecimentos ao Prof. Manoj K. Malhotra, Department Chair do Management Science Department, Moore School of Business da University of South Carolina - USC, Estados Unidos. Seu apoio como orientador em meu programa de Doutorado Sandwich na USC foi fundamental para o refinamento de diversos aspectos relacionados com o tema do estudo e com as definições metodológicas desta tese. Estendo meus agradecimento ao Prof. Jayanth Jayaram, também do Management Science Department, pelos comentários e sugestões de melhoria no escopo da tese.

Aos professores do Departamento de Engenharia Industrial - DEI pelo compartilhamento de experiências e contribuições com seus conhecimentos. Também a todo o pessoal da secretaria do DEI pela amizade e apoio prestados. 
Ao Programa de Pós-Graduação em Engenharia de Produção da COPPE/UFRJ por ter permitido que fossem cursadas disciplinas ministradas por professores do seu quadro docente.

Aos Diretores, Coordenadores e Gerentes de Produção das empresas que, gentilmente, participaram desta pesquisa, contribuindo com suas percepções gerenciais sobre o tema proposto. As suas visões práticas foram essenciais para a condução do estudo empírico.

Ao Professor Ualison Rébula de Oliveira, da AEDB e Estácio de Sá, e ao Coordenador de Projetos do SEBRAE/RJ, Antonio Batista Ribeiro Neto, pela indicação e auxílio no contato e acesso às empresas que compõem a amostra deste estudo.

Aos colegas de curso na PUC-Rio pela amizade e parceria ao longo desses anos no Programa de Pós-Graduação do DEI. Também aos alunos de doutorado do Management Science Department da USC e demais amigos em Columbia, SC, Estados Unidos. Em especial, aos amigos/irmãos paraenses com quem convivi grande parte de minha vida no Rio de Janeiro, Aluisio, Dayvid e Denner Monteiro e Antonio Batista.

À minha querida esposa, Danielle, que tive a felicidade de encontrar no momento certo em minha vida para ser minha companheira, amiga e grande incentivadora.

Aos meus pais, Roque e Osmarina Serrão, e demais familiares em Belém/PA pelo apoio sempre presente, mesmo à distância e pelo incentivo à busca contínua de conhecimentos. Ainda, ao apoio da minha "família carioca" herdada a partir de minha esposa.

À CAPES pelo apoio financeiro concedido ao longo do programa de doutorado na PUC-Rio.

Ao CNPq pelo apoio financeiro durante meu programa de Doutorado Sandwich no Exterior. 


\section{Resumo}

Serrão, Rogério Odivan Brito; Dalcol, Paulo Roberto Tavares. Operacionalização da flexibilidade de manufatura: análise das influências do relacionamento produtor-fornecedor. Rio de Janeiro, 2005. 159p. Tese de Doutorado - Departamento de Engenharia Industrial, Pontifícia Universidade Católica do Rio de Janeiro.

O tema principal desta tese é a operacionalização da flexibilidade de manufatura, a partir de duas abordagens: teórica e empírica. A abordagem teórica visa a discussão, com base em uma extensa revisão da bibliografia, da flexibilidade de manufatura e a sua operacionalização. No tocante à operacionalização, destaca-se a existência de diversos aspectos considerados "indutores", como a tecnologia de processo, métodos de gerenciamento da produção, gerenciamento de recursos humanos, tecnologia de informação, processo de desenvolvimento de produtos e os relacionamentos na cadeia de suprimentos. Dentre esses aspectos, verificou-se a necessidade de aprofundamento na questão que trata dos relacionamentos na cadeia de suprimentos, em especial os relacionamentos produtor-fornecedor. Após o desenvolvimento do referencial teórico sobre os relacionamentos na cadeia de suprimentos, no sentido de apresentar as características gerais e seus efeitos sobre a flexibilidade de manufatura, passou-se a uma análise empírica com o foco no relacionamento produtor-fornecedor. A escolha desse indutor para a condução do estudo empírico deu-se em função da emergência do papel dos fornecedores na definição das estratégias do produtor e da importância das relações entre produtor e fornecedor na criação e desenvolvimento de vantagem competitiva. Além da construção de um referencial teórico - útil para a discussão da operacionalização da flexibilidade de manufatura como fonte de vantagem competitiva e preparação para futuras pesquisas - a tese também atende à demanda por estudos empíricos tão importantes para o avanço das pesquisas sobre o tema. Nesse sentido, buscou-se responder às duas questões de pesquisa: "Como se caracterizam os 
relacionamentos produtor-fornecedor quanto aos aspectos de confiança/comprometimento, compartilhamento de informações, desenvolvimento de fornecedores e desenvolvimento conjunto de produtos nas empresas estudadas?" e “Como se dão os impactos dos aspectos de relacionamento produtor-fornecedor sobre a flexibilidade de manufatura e o desempenho da manufatura nas empresas estudadas?" O estudo envolveu quatro empresas de manufatura em uma investigação da influência de quatro aspectos de relacionamento produtor-fornecedor - c confiança/comprometimento, compartilhamento de informações, desenvolvimento de fornecedores e desenvolvimento conjunto de produtos - sobre três dimensões externas de flexibilidade de manufatura - mix, novos produtos e volume. A análise estendeuse à observação dos efeitos generalizados dessas relações sobre o desempenho da manufatura e dos níveis da flexibilidade de manufatura apresentados pelas empresas estudadas.

\section{Palavras-chave}

Flexibilidade de manufatura; relacionamento produtor-fornecedor; desempenho da manufatura. 


\section{Abstract}

Serrão, Rogério Odivan Brito; Dalcol, Paulo Roberto Tavares (Advisor). Operationalization of manufacturing flexibility: analyzing the influence of the buyer-supplier relationships. Rio de Janeiro, 2005. 159p. Ph.D. Dissertation - Departamento de Engenharia Industrial, Pontifícia Universidade Católica do Rio de Janeiro.

The purpose of this dissertation is to study the operationalization of manufacturing flexibility, focusing on aspects of relationships in the supply chain and their impact on manufacturing flexibility. The research involves four manufacturing companies for studying the influence of four aspects of buyersupplier relationship - trust/commitment, information sharing, supplier development and joint product development - on three external dimensions of manufacturing flexibility - mix, new products and volume. The study also observes the generalized effects of these relationships on manufacturing performance and on the levels of manufacturing flexibility shown by the companies studied. The buyer-supplier relationships are deeply analyzed and their general characteristics and effects on the operationalization of manufacturing flexibility are presented. In addition, the analysis of scope and achievability factors indicated the presence of little explored flexible options, the presence of potential flexibility or the underutilization of flexible resources in the companies studied. Finding ways for operationalizing flexibility must be seen as a priority question to obtain competitive advantage in manufacturing systems. This work shows how the relationships between the buyer and supplier can influence several external dimensions of manufacturing flexibility and, consequently, manufacturing performance.

\section{Keywords}

Manufacturing flexibility; buyer-supplier relationships; manufacturing performance. 


\section{Sumário}

$\begin{array}{ll}\text { Lista de figuras } & 14\end{array}$

$\begin{array}{ll}\text { Lista de quadros } & 16\end{array}$

1 Introdução 19

1.1 Linhas de pesquisa correntes sobre o tema do estudo 20

1.2 Importância do estudo 22

$\begin{array}{lll}1.3 & \text { Objetivos do estudo } & 23\end{array}$

1.4 Metodologia geral $\quad 24$

1.5 Estrutura da tese 25

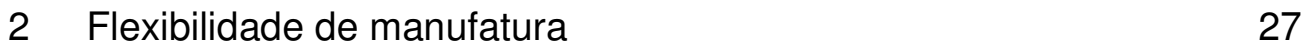

2.1 Necessidade de flexibilidade nos sistemas produtivos 27

2.2 Natureza da flexibilidade 29

2.2.1 Definindo flexibilidade $\quad 30$

2.2.2 Conceito multidimensional 30

2.2.3 Flexibilidade requerida, potencial e real 31

2.2.4 Flexibilidade estática e dinâmica 32

2.2.5 Flexibilidade interna e externa 32

2.3 Dimensões e elementos da flexibilidade 33

2.4 Fatores de escopo e atingimento da flexibilidade 38

3 Operacionalização da flexibilidade de manufatura 40

3.1 Tecnologia de processo 43

3.2 Gerenciamento de recursos humanos 48 
3.3 Processo de desenvolvimento de produtos

3.4 Tecnologia de informação 56

3.5 Técnicas de gerenciamento da produção 59

4 Relacionamentos na cadeia de suprimentos 61

4.1 Sobre o gerenciamento da cadeia de suprimentos 61

4.2 Portfolio de relacionamentos produtor-fornecedor 62

4.3 Tendências nos relacionamentos produtor-fornecedor 64

4.4 Aspectos de relacionamento produtor-fornecedor 67

4.4.1 Confiança/comprometimento 67

4.4.2 Compartilhamento de Informações 69

4.4.3 Desenvolvimento de fornecedores 71

4.4.4 Desenvolvimento conjunto de produtos 75

4.5 A importância da flexibilidade na cadeia de suprimentos 77

4.6 Relacionamentos na cadeia de suprimentos e as dimensões da flexibilidade de manufatura $\quad 79$

5 Projeto de pesquisa para o estudo empírico 81

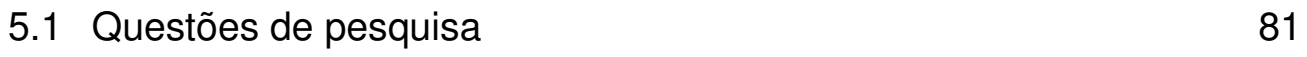

5.2 Desenvolvimento do modelo conceitual 82

5.3 Proposições gerais de pesquisa: visualizando o papel moderador do tipo de relacionamento produtor-fornecedor 84

5.4 Definição de itens de medição 85

5.5 Metodologia de coleta de dados 90

6 Descrição dos casos da pesquisa empírica 92

6.1 Estudo de caso - EMPRESA A 92 
6.1.1 Caracterização dos principais produtos, mercado e fornecedores da EMPRESA A

6.2 Estudo de caso - EMPRESA B

6.2.1 Caracterização dos principais produtos, mercado e fornecedores da EMPRESA B

6.3 Estudo de caso - EMPRESA C

6.3.1 Caracterização dos principais produtos, mercado e fornecedores da EMPRESA C

6.4 Estudo de caso - EMPRESA D

6.4.1 Caracterização dos principais produtos, mercado e fornecedores da EMPRESA D

7 Aspectos de relacionamento produtor-fornecedor - Primeira questão de pesquisa

7.1 Características gerais de relacionamento produtor-fornecedor na EMPRESA A

7.1.1 Confiança/comprometimento do fornecedor na EMPRESA A

7.1.2 Desenvolvimento de fornecedores na EMPRESA A

7.1.3 Compartilhamento de informações na EMPRESA A

7.1.4 Desenvolvimento conjunto de produtos na EMPRESA A

7.2 Características gerais de relacionamento produtor-fornecedor na EMPRESA B

7.2.1 Confiança/comprometimento do fornecedor na EMPRESA B

7.2.2 Desenvolvimento de fornecedores na EMPRESA B

7.2.3 Compartilhamento de informações na EMPRESA B

7.2.4 Desenvolvimento conjunto de produtos na EMPRESA B

7.3 Características gerais de relacionamento produtor-fornecedor na EMPRESA C

7.3.1 Confiança/comprometimento do fornecedor na EMPRESA C 
7.4 Características gerais de relacionamento produtor-fornecedor na EMPRESA D

7.4.1 Confiança/comprometimento do fornecedor na EMPRESA D 121

7.4.2 Desenvolvimento de fornecedores na EMPRESA D 122

7.4.3 Compartilhamento de informações na EMPRESA D 122

7.4.4 Desenvolvimento conjunto de produtos na EMPRESA D

7.5 Comparação entre os aspectos de relacionamento produtorfornecedor

8 Impactos do relacionamento produtor-fornecedor sobre a flexibilidade e o desempenho da manufatura - segunda questão de pesquisa 130

8.1 Influência dos aspectos de relacionamento produtor-fornecedor sobre a flexibilidade de manufatura nas empresas estudadas

8.2 Escopo e atingimento da flexibilidade de manufatura nas empresas estudadas

8.3 Influência do nível de flexibilidade de manufatura sobre indicadores de desempenho da manufatura

9 Conclusões e considerações finais

10 Referências bibliográficas

11 Anexo 


\section{Lista de figuras}

Figura 1 - Hierarquia das dimensões da flexibilidade

Figura 2 - Caracterização da flexibilidade de manufatura por dimensões e elementos

Figura 3 - Composição dos elementos da flexibilidade de manufatura 37

Figura 4 - Fatores de escopo e atingimento da flexibilidade de manufatura

Figura 5 - Elementos indutores da flexibilidade de manufatura

Figura 6 - Portfolio de relacionamentos produtor-fornecedor.

Figura 7 - Gerenciamento do portfolio de relacionamentos produtorfornecedor

Figura 8 - Progressão de estratégias de desenvolvimento e melhoria de desempenho de fornecedores

Figura 9 - Integração do fornecedor nos estágios de desenvolvimento de produtos

Figura 10 - Características favoráveis a integração de fornecedores em diferentes estágios de desenvolvimento dos produtos

Figura 11 - Evolução dos custos e da flexibilidade ao longo dos estágios de desenvolvimento de produtos

Figura 12 - A flexibilidade associada às relações entre plantas de manufatura

Figura 13 - Modelo de análise para os relacionamentos produtorfornecedor e a flexibilidade de manufatura

Figura 14 - Aspectos de relacionamento na cadeia de suprimentos e os tipos de relacionamento produtor-fornecedor

Figura 15 - Intensidade das características dos principais produtos da EMPRESA A

Figura 16 - Intensidade das características do mercado da 
Figura 17 - Intensidade das características dos principais fornecedores da EMPRESA A

Figura 18 - Intensidade das características principais produtos da

EMPRESA B

Figura 19 - Intensidade das características do mercado da

EMPRESA B

Figura 20 - Intensidade das características dos principais fornecedores da EMPRESA B

Figura 21 - Intensidade das características dos principais produtos

da EMPRESA C

Figura 22 - Intensidade das características do mercado da

EMPRESA C

Figura 23 - Intensidade das características dos principais fornecedores da EMPRESA C

Figura 24 - Intensidade das características dos principais produtos da EMPRESA D

Figura 25 - Intensidade das características do mercado da EMPRESA D

Figura 26 - Intensidade das características dos principais fornecedores da EMPRESA D

Figura 27 - Influência dos aspectos de relacionamento produtorfornecedor sobre a flexibilidade de manufatura

Figura 28 - Escopo e atingimento da flexibilidade de manufatura nas empresas estudadas

Figura 29 - Influência do nível de flexibilidade de manufatura sobre indicadores de desempenho da manufatura na EMPRESA A

Figura 30 - Influência do nível de flexibilidade de manufatura sobre indicadores de desempenho da manufatura na EMPRESA B

Figura 31 - Influência do nível de flexibilidade de manufatura sobre indicadores de desempenho da manufatura na EMPRESA C

Figura 32 - Influência do nível de flexibilidade de manufatura sobre indicadores de desempenho da manufatura na EMPRESA D 


\section{Lista de quadros}

Quadro 1 - Razões para a necessidade de flexibilidade. 29

Quadro 2 - Algumas definições propostas para a flexibilidade 31

Quadro 3 - Definições das dimensões de flexibilidade de manufatura 34

Quadro 4 - Caracterização dos elementos da flexibilidade de manufatura

Quadro 5-Referencial bibliográfico para os indutores de flexibilidade 42

Quadro 6 - Exemplos de investimentos específicos feitos por parceiros em um relacionamento produtor-fornecedor

Quadro 7 - Comparação entre os modelos de relacionamento adversário e cooperativo na indústria automotiva

Quadro 8 - Vantagens e desvantagens para os PRODUTORES em relacionamentos cooperativos produtor-fornecedor.

Quadro 9 - Vantagens e desvantagens para os FORNECEDORES em relacionamentos cooperativos produtor-fornecedor.

Quadro 10 - Diferenciação entre as abordagens reativa e estratégica no desenvolvimento de fornecedores

Quadro 11 - Visão generalizada das etapas do processo evolucionário no desenvolvimento de fornecedores

Quadro 12 - Aspectos de relacionamento na cadeia de suprimentos e as dimensões externas da flexibilidade de manufatura

Quadro 13 - Itens de referência do perfil contextual dos relacionamentos produtor-fornecedor

Quadro 14 - Itens de referência para o desempenho da manufatura

Quadro 15 - Itens de referência dos aspectos de relacionamento

produtor-fornecedor: confiança/comprometimento

Quadro 16 - Itens de referência dos aspectos de relacionamento produtor-fornecedor: compartilhamento de informações

Quadro 17 - Itens de referência dos aspectos de relacionamento produtor-fornecedor: desenvolvimento de fornecedores 
Quadro 18 - Itens de referência dos aspectos de relacionamento produtor-fornecedor: desenvolvimento conjunto de produtos $\quad 89$

Quadro 19 - Informantes-chave nas empresas estudadas 91

Quadro 20 - Síntese de informações características das empresas estudadas

Quadro 21 - Confiança/comprometimento do fornecedor no relacionamento nas empresas estudadas

Quadro 22 - Desenvolvimento de fornecedores no relacionamento nas empresas estudadas

Quadro 23 - Compartilhamento de informações no relacionamento nas empresas estudadas

Quadro 24 - Desenvolvimento conjunto de produtos no relacionamento nas empresas estudadas 
"Nothing is quite so practical as a good theory"

Andrew $\mathrm{H}$. van de ven The Academy of Management Review, 14, 4, 1989. 